\title{
A note on existence of infinitely many periodic solutions for second-order nonlinear difference systems
}

Qin Jiang and Sheng $\mathrm{Ma}^{*}$

"Correspondence:

masheng666@126.com Department of Mathematics,

Huanggang Normal University, Hubei, 438000, China

\begin{abstract}
The paper deals with the existence of infinitely many periodic solutions for second-order nonlinear difference systems. A variational approach is applied using the saddle point theorem.
\end{abstract}

Keywords: second-order difference systems; infinitely many periodic solutions; variational methods; saddle point theorem

\section{Introduction and statement of main results}

The interest in the second-order difference systems

$$
\left\{\begin{array}{l}
\triangle^{2} u(t-1)+\nabla F(t, u(t))=0, \quad t \in \mathbb{Z}[1, T] \\
u(0)=u(T),
\end{array}\right.
$$

has been aroused, where $\Delta u(t)=u(t+1)-u(t), \Delta^{2} u(t)=\Delta(\Delta u(t)), \nabla F(t, x)=\frac{\partial F(t, x)}{\partial x}, T$ is a positive integer, and $\mathbb{Z}$ and $R$ denote the set of integers and the set of real numbers, respectively, and $\mathbb{Z}[a, b]=\{a, a+1, \ldots, b\}$, for $a, b \in \mathbb{Z}$ and $a \leq b$. Assume that $F(t, x)$ is $T$-periodic in $t$ for all $x \in R^{N}$ and $F(t, x) \in C^{1}\left(\mathbb{Z} \times R^{N}, R\right)$.

In 2003, Yu and Guo [1-3] established a variational structure and variational methods to study discrete Hamiltonian systems and obtain the solvability condition of a periodic solution for discrete systems, based on operator theory. Since then more and more authors have contributed to study second-order discrete Hamiltonian systems, with an effective tool named the critical point theory, and one obtained many interesting results [4-11]. In [8], with operator theory, Xue and Tang constructed a variational setting unlike the one in [1] to study the second-order superquadratic discrete Hamiltonian systems (1) and obtained the existence of periodic solutions. This result generalized the one in [4]. In [7], Xue and Tang obtained the existence of one periodic solution of systems (1) under the hypothesis there exist $M_{1}>0, M_{2}>0$ and $0 \leq \alpha<1$ such that

$$
|\nabla F(t, x)| \leq M_{1}|x|^{\alpha}+M_{2}
$$

(c) Jiang and Ma 2016. This article is distributed under the terms of the Creative Commons Attribution 4.0 International License (http://creativecommons.org/licenses/by/4.0/), which permits unrestricted use, distribution, and reproduction in any medium, provided you give appropriate credit to the original author(s) and the source, provide a link to the Creative Commons license, and indicate if changes were made. 
for all $(t, x) \in \mathbb{Z}[1, T] \times R^{N}$, and the condition

$$
|x|^{-2 \alpha} \sum_{t=1}^{T} F(t, x) \rightarrow+\infty \quad \text { as }|x| \rightarrow \infty
$$

Subsequently, in [9], Yan, Wu and Tang extended these results in [7] to the case that $F(t, x)$ is $T_{i}$-periodic in $x_{i}$ and obtained the existence of multiple periodic solutions, where $x_{i}$ is the $i$ th component of $x=\left(x_{1}, x_{2}, \ldots, x_{N}\right), i \in[1, N]$. Especially in [12], Che and Xue obtained the existence of infinitely many periodic solutions for systems in the case that:

(F1) there exist $f, g: \mathbb{Z}[0, T] \rightarrow R^{+}$and $\alpha \in[0,1)$ such that

$$
|\nabla F(t, x)| \leq f(t)|x|^{\alpha}+g(t) \quad \text { for all }(t, x) \in \mathbb{Z}[0, T] \times R^{N},
$$

and a suitable oscillating behaviour at infinity,

(F2) $\liminf _{r \rightarrow \infty} \sup _{x \in R^{N},|x|=r}|x|^{-2 \alpha} \sum_{t=0}^{T} F(t, x)=-\infty$,

(H3) $\limsup \sup _{r \rightarrow \infty} \inf _{x \in R^{N},|x|=r} \sum_{t=0}^{T} F(t, x)=+\infty$.

Consequently, it is natural to ask if infinitely many solutions still exist when $\alpha=1$. With the fact that $\alpha=1,(\mathrm{~F} 1)$ and (F2), respectively, change to the linearly bounded gradient condition:

$\left(\mathrm{F} 1^{\prime}\right)$ there exist $f, g: \mathbb{Z}[0, T] \rightarrow R^{+}$such that

$$
|\nabla F(t, x)| \leq f(t)|x|+g(t) \quad \text { for all }(t, x) \in \mathbb{Z}[0, T] \times R^{N},
$$

and the condition

$\left(\mathrm{F}^{\prime}\right) \liminf _{r \rightarrow \infty} \sup _{x \in R^{N},|x|=r}|x|^{-2} \sum_{t=0}^{T} F(t, x)=-\infty$.

However, similarly to what was pointed in [13], (F2') does not hold if $\sum_{t=0}^{T} f(t)$ is bounded. Therefore, it is necessary to improve condition $\left(\mathrm{F}^{\prime}\right)$. Inspired by $[7,12,13]$, in this paper, we will use minmax methods to further study systems (1) under the following assumptions:

(H1) there exist $f, g: \mathbb{Z}[0, T] \rightarrow R^{+}$with $\sum_{t=0}^{T} f(t)<\frac{\lambda_{1}}{2}$ such that

$$
|\nabla F(t, x)| \leq f(t)|x|+g(t) \quad \text { for all }(t, x) \in \mathbb{Z}[0, T] \times R^{N},
$$

(H2) $\liminf _{r \rightarrow+\infty} \sup _{x \in R^{N},|x|=r}|x|^{-2} \sum_{t=0}^{T} F(t, x)<-\frac{4 \sum_{t=0}^{T} f^{2}(t)}{\lambda_{1}}$, where $\lambda_{k}=2-2 \cos \frac{2 k \pi}{T}$ satisfy the eigenvalue problem

$$
-\triangle^{2} u(t-1)=\lambda_{k} u(t), \quad k \in \mathbb{Z}\left[0,\left[\frac{T}{2}\right]\right],
$$

and we note

$$
0=\lambda_{0}<\lambda_{1}<\cdots<\lambda_{\left[\frac{T}{2}\right]} \leq 4
$$

The main result on the existence of infinitely many periodic solutions of systems (1) is obtained. The details are described. 
Theorem 1.1 Under the hypotheses of (H1), (H2), and (H3):

(a) discrete systems (1) have a sequence $\left\{u_{n}\right\}$ of solutions such that $\left\{u_{n}\right\}$ is a critical point of the functional $\varphi$ and $\varphi\left(u_{n}\right) \rightarrow+\infty$ as $n \rightarrow \infty$;

(b) discrete systems (1) have a sequence $\left\{u_{n}^{*}\right\}$ of solutions such that $\left\{u_{n}^{*}\right\}$ is a local minimizer of $\varphi$ and $\varphi\left(u_{n}^{*}\right) \rightarrow-\infty$ as $n \rightarrow \infty$,

where the variational functional $\varphi$ is

$$
\varphi(u)=\frac{1}{2} \sum_{t=0}^{T}|\Delta u(t)|^{2}-\sum_{t=0}^{T} F(t, u(t)),
$$

on Hilbert space $H_{T}$ defined by

$$
H_{T}=\left\{u: \mathbb{Z} \rightarrow R^{N} \mid u(t)=u(t+T), t \in \mathbb{Z}\right\},
$$

with the inner product and the norm, respectively, written as

$$
\langle u, v\rangle=\sum_{t=0}^{T}(u(t), v(t)), \quad \forall u, v \in H_{T}
$$

and

$$
\|u\|=\left(\sum_{t=0}^{T}|u(t)|^{2}\right)^{\frac{1}{2}} .
$$

Remark 1.2 As pointed out in [12], the nonlinearity potential F does not need the symmetry condition in the paper. Moreover, Theorem 1.1 is a complement to and development of Theorem 1.1 in [12] corresponding to $\alpha=1$.

\section{Proof of main result}

For all $u \in H_{T},\|u\|_{\infty}=\sup _{t \in \mathbb{Z}[0, T]}|u(t)|$ is defined. It is obvious that

$$
\|u\|_{\infty} \leq\|u\|=\left(\sum_{t=0}^{T}|u(t)|^{2}\right)^{\frac{1}{2}} .
$$

By the definition of $H_{T}, H_{T}$ is finite dimensional. By $(\mathrm{H} 1)$, one gets $\varphi \in C^{1}\left(H_{T}, R\right)$ and

$$
\left\langle\varphi^{\prime}(u), v\right\rangle=\sum_{t=0}^{T}(\Delta u(t), \Delta v(t))-\sum_{t=0}^{T}(\nabla F(t, u(t)), v(t)),
$$

for all $u, v \in H_{T}$.

Subsequently, an important lemma in [8] is shown for the reader's convenience. The lemma is constructed in a variational setting, with the operator theory, unlike the one in [1]. Details can be found in [8].

Lemma 1 ([8]) Let $N_{k}$ be a subspace of $H_{T}$ written as

$$
N_{k}:=\left\{u \in H_{T} \mid-\triangle^{2} u(t-1)=\lambda_{k} u(t)\right\},
$$

where $\lambda_{k}=2-2 \cos \frac{2 k \pi}{T}, k \in \mathbb{Z}\left[0,\left[\frac{T}{2}\right]\right]$. Then one has the results as follows: 
(i) $N_{k} \perp N_{j}, k \neq j, k, j \in \mathbb{Z}\left[0,\left[\frac{T}{2}\right]\right]$.

(ii) $H_{T}=\bigoplus_{k=0}^{[T / 2]} N_{k}$.

Let $V=N_{0}$ and $W=N_{0}^{\perp}=\bigoplus_{k=1}^{[T / 2]} N_{k}$. Via Lemma 2 in [8], we have

$$
H_{T}=N_{0} \oplus N_{0}^{\perp}=V \oplus W
$$

and

$$
\sum_{t=0}^{T}|\Delta u(t)|^{2} \geq \lambda_{1}\|u\|^{2}, \quad \forall u \in W=N_{0}^{\perp} .
$$

From the definition of $N_{0}$, one gets $u(t)=u(0)=C \in R^{N}$, for all $u \in N_{0}$ and $t \in \mathbb{Z}[0, T]$. By Lemma 1 , one rewrites $u$ as $u=\bar{u}+\tilde{u} \in V \oplus W=N_{0} \oplus N_{0}^{\perp}$, where

$$
\bar{u}=\frac{1}{T} \sum_{t=0}^{T} u(t)
$$

From the fact that

$$
\begin{aligned}
\|u\| & =\left(\sum_{t=0}^{T}|u(t)|^{2}\right)^{\frac{1}{2}}=\left(\sum_{t=0}^{T}|\bar{u}+\tilde{u}(t)|^{2}\right)^{\frac{1}{2}} \\
& =\left(\sum_{t=0}^{T}\langle\bar{u}+\tilde{u}, \bar{u}+\tilde{u}\rangle\right)^{\frac{1}{2}} \\
& =\left(\sum_{t=0}^{T}\left(|\bar{u}|^{2}+|\tilde{u}(t)|^{2}\right)\right)^{\frac{1}{2}}=\left(T|\bar{u}|^{2}+\|\tilde{u}\|^{2}\right)^{\frac{1}{2}},
\end{aligned}
$$

one has

$$
\|u\| \leq \sqrt{T+1}\left(|\bar{u}|^{2}+\|\tilde{u}\|^{2}\right)^{\frac{1}{2}} \quad \text { and } \quad\|u\| \geq\left(|\bar{u}|^{2}+\|\tilde{u}\|^{2}\right)^{\frac{1}{2}} .
$$

Thus one obtains that $\|u\| \rightarrow \infty$ if and only if $\left(|\bar{u}|^{2}+\|\tilde{u}\|^{2}\right)^{\frac{1}{2}} \rightarrow \infty$.

Proof of Theorem 1.1 The proof of Theorem 1.1 relies on a minimax theorem (Corollary 4.3) in [14]. We complete the proof with a series of statements below.

Step 1 , we claim that $\varphi$ is coercive in the subspace $W$.

Due to (H1), there exists a constant $C_{1}$ satisfying the following inequality:

$$
\begin{aligned}
|F(t, x)| & \leq\left|\int_{0}^{1}(\nabla F(t, s x), x) d s\right|+|F(t, 0)| \\
& \leq \int_{0}^{1}|\nabla F(t, s x)||x| d s+C_{1} \leq \frac{f(t)}{2}|x|^{2}+g(t)|x|+C_{1}
\end{aligned}
$$


for all $t \in \mathbb{Z}[0, T]$ and $x \in R^{N}$. Hence, using the Hölder inequality, (2), (3), and (4), for all $u \in W$, one derives

$$
\begin{aligned}
\varphi(u) & =\frac{1}{2} \sum_{t=0}^{T}|\Delta u(t)|^{2}-\sum_{t=0}^{T} F(t, u) \\
& \geq \frac{1}{2} \lambda_{1}\|u\|^{2}-\sum_{t=0}^{T}\left(\frac{1}{2} f(t)|u(t)|^{2}+g(t)|u(t)|+C_{1}\right) \\
& \geq \frac{1}{2} \lambda_{1}\|u\|^{2}-\|u\|_{\infty}^{2} \sum_{t=0}^{T} \frac{1}{2} f(t)-\|u\|_{\infty} \sum_{t=0}^{T} g(t)-C_{1} T \\
& \geq \frac{1}{2} \lambda_{1}\|u\|^{2}-\|u\|^{2} \frac{1}{2} \sum_{t=0}^{T} f(t)-\|u\| \sum_{t=0}^{T} g(t)-C_{1} T \\
& =\left(\frac{1}{2} \lambda_{1}-\frac{1}{2} \sum_{t=0}^{T} f(t)\right)\|u\|^{2}-\|u\| \sum_{t=0}^{T} g(t)-C_{1} T .
\end{aligned}
$$

Combining this with the fact $\sum_{t=0}^{T} f(t)<\frac{1}{2} \lambda_{1}$, one deduces $\lim _{\|u\| \rightarrow+\infty} \varphi(u)=+\infty$.

Step 2 , we claim that there are positive sequences $\left\{a_{n}\right\},\left\{b_{m}\right\}$ satisfying

(c) $\lim _{n \rightarrow \infty} a_{n}=+\infty$ and $\lim _{n \rightarrow \infty} \sup _{u \in V,\|u\|=a_{n}} \varphi(u)=-\infty$,

(d) $\lim _{m \rightarrow \infty} b_{m}=+\infty$ and $\lim _{m \rightarrow \infty} \inf _{u \in H_{b_{m}}} \varphi(u)=+\infty$,

where $H_{b_{m}}=\left\{u \in V,\|u\|=b_{m}\right\} \oplus W$.

The detailed proof of (c) can be founded in [12]. On the other hand, by (H2), one can take a constant

$$
a>\frac{8}{\lambda_{1}}
$$

Thus one gets

$$
\liminf _{r \rightarrow \infty} \sup _{x \in R^{N},|x|=r}|x|^{-2} \sum_{t=0}^{T} F(t, x)<-\frac{a}{2} \sum_{t=0}^{T} f^{2}(t) .
$$

For any $u \in H_{b_{m}}$, rewritten $u=\tilde{u}+\bar{u}$, where $\tilde{u} \in W$ and $\bar{u} \in V$, by the Hölder inequality, (H1), and (H2), one has

$$
\begin{aligned}
& \left|\sum_{t=0}^{T}(F(t, u(t))-F(t, \bar{u}))\right| \\
& \quad \leq \sum_{t=0}^{T} \int_{0}^{1}|(\nabla F(t, \bar{u}+s \tilde{u}(t)), \tilde{u}(t))| d s \\
& \quad \leq \sum_{t=0}^{T} \int_{0}^{1}(f(t)|\bar{u}+s \tilde{u}(t)|+g(t)) \cdot|\tilde{u}(t)| d s \\
& \quad \leq \sum_{t=0}^{T} f(t)\left(|\bar{u}|+\frac{1}{2}|\tilde{u}(t)|\right)|\tilde{u}(t)|+\sum_{t=0}^{T} g(t)|\tilde{u}(t)|
\end{aligned}
$$




$$
\begin{aligned}
& \leq|\bar{u}|\left(\sum_{t=0}^{T} f^{2}(t)\right)^{\frac{1}{2}}\left(\sum_{t=0}^{T}|\tilde{u}(t)|^{2}\right)^{\frac{1}{2}}+\frac{1}{2}\|\tilde{u}\|_{\infty}^{2} \sum_{t=0}^{T} f(t)+\|\tilde{u}\|_{\infty} \sum_{t=0}^{T} g(t) \\
& \leq \frac{1}{2 a}\|\tilde{u}\|^{2}+\frac{a}{2} \sum_{t=0}^{T} f^{2}(t)|\bar{u}|^{2}+\frac{1}{2} \sum_{t=0}^{T} f(t)\|\tilde{u}\|^{2}+\|\tilde{u}\| \sum_{t=0}^{T} g(t) \\
& \leq\left(\frac{1}{2 a}+\frac{1}{2} \sum_{t=0}^{T} f(t)\right)\|\tilde{u}\|^{2}+\frac{a}{2} \sum_{t=0}^{T} f^{2}(t)|\bar{u}|^{2}+\|\tilde{u}\| \sum_{t=0}^{T} g(t) .
\end{aligned}
$$

Hence, for all $u \in H_{b_{m}}$, it follows from inequalities (3) and (6) that

$$
\begin{aligned}
\varphi(u)= & \frac{1}{2} \sum_{t=0}^{T}|\Delta u(t)|^{2}-\sum_{t=0}^{T} F(t, u(t)) \\
= & \frac{1}{2} \sum_{t=0}^{T}|\Delta \tilde{u}(t)|^{2}-\sum_{t=0}^{T}(F(t, u(t))-F(t, \bar{u}))-\sum_{t=0}^{T} F(t, \bar{u}) \\
\geq & \frac{1}{2} \lambda_{1}\|\tilde{u}\|^{2}-\left(\frac{1}{2 a}+\frac{1}{2} \sum_{t=0}^{T} f(t)\right)\|\tilde{u}\|^{2}-\frac{a}{2} \sum_{t=0}^{T} f^{2}(t)|\bar{u}|^{2} \\
& -\|\tilde{u}\| \sum_{t=0}^{T} g(t)-\sum_{t=0}^{T} F(t, \bar{u}) \\
= & \left.\frac{\lambda_{1}}{2}-\frac{1}{2 a}-\frac{1}{2} \sum_{t=0}^{T} f(t)\right)\|\tilde{u}\|^{2}-\|\tilde{u}\| \sum_{t=0}^{T} g(t) \\
& -|\bar{u}|^{2}\left(\frac{\sum_{t=0}^{T} F(t, \bar{u})}{|\bar{u}|^{2}}+\frac{a}{2} \sum_{t=0}^{T} f^{2}(t)\right) .
\end{aligned}
$$

By $\sum_{t=0}^{T} f(t)<\frac{\lambda_{1}}{2}$ and $a>\frac{8}{\lambda_{1}}$,

$$
\frac{\lambda_{1}}{2}-\frac{1}{2 a}-\frac{1}{2} \sum_{t=0}^{T} f(t)>0
$$

is verified. By (5), (7), and the fact $\|u\| \rightarrow \infty$ if and only if $\left(|\bar{u}|^{2}+\|\tilde{u}\|^{2}\right)^{\frac{1}{2}} \rightarrow \infty$, the conclusion $(\mathrm{d})$ is achieved.

Now we have a family of maps $\Gamma_{n}$ expressed as

$$
\Gamma_{n}=\left\{\gamma \in C\left(B_{a_{n}}, H_{T}\right)|\gamma|_{\partial B_{a_{n}}}=\left.\operatorname{Id}\right|_{\partial B_{a_{n}}}\right\}
$$

and minimax values $c_{n}$ formulated as

$$
c_{n}=\inf _{\gamma \in \Gamma_{n}} \max _{u \in B_{a_{n}}} \varphi(\gamma(u))
$$

for each $n$, where $B_{a_{n}}$ is a ball in $\mathrm{V}$ and $a_{n}$ is the radius of $B_{a_{n}}$. One gets

$$
\gamma\left(B_{a_{n}}\right) \cap W \neq \emptyset
$$

for any $\gamma \in \Gamma_{n}$ from Theorem 4.6 in [14]. 
Step 3, we claim that, for sufficiently large $n$, there exist sequences $\left\{\gamma_{k}\right\} \subset \Gamma_{n}$ and $\left\{v_{k}\right\}$ in $H_{T}$, respectively, satisfying

$$
\begin{aligned}
& \max _{u \in B_{a_{n}}} \varphi\left(\gamma_{k}(u)\right) \rightarrow c_{n}, \\
& \varphi\left(v_{k}\right) \rightarrow c_{n}, \quad \varphi^{\prime}\left(v_{k}\right) \rightarrow 0, \quad \operatorname{dist}\left(v_{k}, \gamma_{k}\left(B_{a_{n}}\right)\right) \rightarrow 0 \quad \text { as } k \rightarrow \infty .
\end{aligned}
$$

By Step 1, we know $\varphi(u) \rightarrow+\infty$ as $\|u\| \rightarrow+\infty, u \in W$. Therefore there exists a constant $C_{2}$ satisfying

$$
\max _{u \in B_{a_{n}}} \varphi(\gamma(u)) \geq \inf _{u \in W} \varphi(u) \geq C_{2} .
$$

Furthermore, one has

$$
c_{n} \geq \inf _{u \in W} \varphi(u) \geq C_{2},
$$

for sufficiently large $n$. By the fact $\gamma\left(B_{a_{n}}\right) \cap W \neq \emptyset$ and the conclusion of Step 2, one obtains

$$
c_{n}>\max _{u \in \partial B_{a_{n}}} \varphi(u)
$$

for sufficiently large $n$. Therefore, for a fixed $n$, this claim is proved from Theorem 4.3 and Corollary 4.3 in [14].

Step 4, we draw the conclusion that the sequence $\left\{v_{k}\right\}$ is bounded in $H_{T}$.

For sufficiently large $k$, by (8), one has

$$
c_{n} \leq \max _{u \in B_{a_{n}}} \varphi\left(\gamma_{k}(u)\right) \leq c_{n}+1 .
$$

We choose $w_{k} \in \gamma_{k}\left(B_{a_{n}}\right)$ satisfying

$$
\left\|v_{k}-w_{k}\right\| \leq 1
$$

From the conclusion (d) of Step 2, for a fixed $n$, a sufficiently large $m$ exists, rendering the formula

$$
b_{m}>a_{n} \text { and } \inf _{u \in H_{b_{m}}} \varphi(u)>c_{n}+1 .
$$

These inequalities imply that $\gamma_{k}\left(B_{a_{n}}\right) \cap H_{b_{m}}=\emptyset$ for each $k$. We now write $w_{k}=\bar{w}_{k}+\tilde{w}_{k}$, where $\bar{w}_{k} \in V$ and $\tilde{w}_{k} \in W$. Then one has

$$
\left\|\bar{w}_{k}\right\|<b_{m}
$$

for each $k$. Moreover, by (2), (3), (4), and (10), one gets

$$
\begin{aligned}
1+c_{n} & \geq \varphi\left(w_{k}\right)=\frac{1}{2} \sum_{t=0}^{T}\left|\Delta w_{k}(t)\right|^{2}-\sum_{t=0}^{T} F\left(t, w_{k}(t)\right) \\
& \geq \frac{1}{2} \lambda_{1}\left\|\tilde{w}_{k}\right\|^{2}-\sum_{t=0}^{T}\left(\frac{1}{2} f(t)\left|w_{k}(t)\right|^{2}+g(t)\left|w_{k}(t)\right|+C_{1}\right)
\end{aligned}
$$




$$
\begin{aligned}
\geq & \frac{1}{2} \lambda_{1}\left\|\tilde{w}_{k}\right\|^{2}-\sum_{t=0}^{T} f(t)\left[\left|\bar{w}_{k}\right|^{2}+\left|\tilde{w}_{k}(t)\right|^{2}\right]-\sum_{t=0}^{T} g(t)\left(\left|\bar{w}_{k}\right|+\left|\tilde{w}_{k}(t)\right|\right)-C_{1} T \\
\geq & \frac{1}{2} \lambda_{1}\left\|\tilde{w}_{k}\right\|^{2}-\left\|\tilde{w}_{k}\right\|_{\infty}^{2} \sum_{t=0}^{T} f(t)-\left\|\bar{w}_{k}\right\|^{2} \sum_{t=0}^{T} f(t) \\
& -\left\|\tilde{w}_{k}\right\|_{\infty} \sum_{t=0}^{T} g(t)-\left\|\bar{w}_{k}\right\| \sum_{t=0}^{T} g(t)-C_{1} T \\
\geq & \frac{1}{2} \lambda_{1}\left\|\tilde{w}_{k}\right\|^{2}-\left\|\tilde{w}_{k}\right\|^{2} \sum_{t=0}^{T} f(t)-b_{m}^{2} \sum_{t=0}^{T} f(t) \\
& -\left\|\tilde{w}_{k}\right\| \sum_{t=0}^{T} g(t)-b_{m} \sum_{t=0}^{T} g(t)-C_{1} T \\
= & \left(\frac{\lambda_{1}}{2}-\sum_{t=0}^{T} f(t)\right)\left\|\tilde{w}_{k}\right\|^{2}-\left\|\tilde{w}_{k}\right\| \sum_{t=0}^{T} g(t)-b_{m}^{2} \sum_{t=0}^{T} f(t)-b_{m} \sum_{t=0}^{T} g(t)-C_{1} T .
\end{aligned}
$$

We can combine equation (11) and the fact that $\sum_{t=0}^{T} f(t)<\frac{\lambda_{1}}{2},\left\|\tilde{w}_{k}\right\|$ is bounded. Thus, by combining (10) and the fact that $\left\|w_{k}\right\|=\left(T\left|\bar{w}_{k}\right|^{2}+\left\|\tilde{w}_{k}\right\|^{2}\right)^{\frac{1}{2}},\left\{w_{k}\right\}$ is bounded. Then $\left\{v_{k}\right\}$ is bounded in $H_{T}$ via (9). The conclusion is proved.

Step 5 , we claim that $c_{n}$ is a critical value of $\varphi$.

Since $\left\{v_{k}\right\}$ is bounded and $H_{T}$ is finite dimensional space, $\left\{v_{k}\right\}$ contains a convergent subsequence that is still denoted as $\left\{v_{k}\right\}$ for convenience, meeting

$$
\lim _{k \rightarrow \infty} v_{k}=u_{n}
$$

Then, by (8), one has

$$
\varphi\left(u_{n}\right)=c_{n} \quad \text { and } \quad \varphi^{\prime}\left(u_{n}\right)=0 .
$$

Thus $\varphi$ has a critical point $u_{n}$.

We prove part (a) of Theorem 1.1. One chooses sufficiently large $n$ satisfying $a_{n}>b_{m}$, then one has $\gamma\left(B_{a_{n}}\right) \cap H_{b_{m}} \neq \emptyset$ for any $\gamma \in \Gamma_{n}$. It follows that

$$
\max _{u \in B_{a_{n}}} \varphi(\gamma(u)) \geq \inf _{u \in H_{b_{m}}} \varphi(u)
$$

With this and the conclusion (d) of Step 2,

$$
\lim _{n \rightarrow \infty} c_{n}=+\infty
$$

is implied. Part (a) of Theorem 1.1 is proved.

A follow-up is to prove part (b) in Theorem 1.1. For a given $m$, let $P_{m}$ be a subset of $H_{T}$, where

$$
P_{m}=\left\{u=\bar{u}+\tilde{u} \in H_{T} \mid \bar{u} \in V,\|\bar{u}\| \leq b_{m}, \tilde{u} \in W\right\} .
$$


For all $u \in P_{m}$, similar to (11), one obtains

$$
\begin{aligned}
\varphi(u) & =\frac{1}{2} \sum_{t=0}^{T}|\triangle u(t)|^{2}-\sum_{t=0}^{T} F(t, u(t)) \\
& \geq \frac{1}{2} \lambda_{1}\|\tilde{u}\|^{2}-\sum_{t=0}^{T}\left(\frac{1}{2} f(t)|u(t)|^{2}+g(t)|u(t)|+C_{1}\right) \\
& \geq\left(\frac{\lambda_{1}}{2}-\sum_{t=0}^{T} f(t)\right)\|\tilde{u}\|^{2}-\|\tilde{u}\| \sum_{t=0}^{T} g(t)-b_{m}^{2} \sum_{t=0}^{T} f(t)-b_{m} \sum_{t=0}^{T} g(t)-C_{1} T .
\end{aligned}
$$

Due to (12), $\varphi$ is bounded below on $P_{m}$. Take

$$
\mu_{m}=\inf _{u \in P_{m}} \varphi(u)
$$

and a sequence $\left\{u_{k}\right\} \subset P_{m}$, satisfying

$$
\varphi\left(u_{k}\right) \rightarrow \mu_{m} \quad \text { as } k \rightarrow \infty
$$

Similar to the proof of the boundedness of $\left\{w_{k}\right\}$ in Step 4, $\left\{u_{k}\right\}$ is bounded in $H_{T}$ via (12). Then $\left\{u_{k}\right\}$ contains a convergent subsequence that is still denoted $\left\{u_{k}\right\}$ for convenience, satisfying

$$
u_{k} \rightarrow u_{m}^{*} \quad \text { weakly in } H_{T}, \quad \text { as } k \rightarrow \infty \text {. }
$$

Noting that $P_{m}$ is convex and closed in $H_{T}$, one has $u_{m}^{*} \in P_{m}$. Moreover, in view of the weakly lower semi-continuity of $\varphi$, one has

$$
\mu_{m}=\lim _{k \rightarrow \infty} \varphi\left(u_{k}\right) \geq \varphi\left(u_{m}^{*}\right)
$$

and

$$
\mu_{m}=\varphi\left(u_{m}^{*}\right)
$$

Next, we draw the conclusion that $u_{m}^{*}$ is an interior point of $P_{m}$. Thus $u_{m}^{*}$ is a critical point of $\varphi$.

Taking

$$
u_{m}^{*}=\bar{u}_{m}^{*}+\tilde{u}_{m}^{*}
$$

where $\bar{u}_{m}^{*} \in V$, $\tilde{u}_{m}^{*} \in W$. If $a_{n}<b_{m}$, one has $\partial B_{a_{n}} \subset P_{m}$, which implies that

$$
\varphi\left(u_{m}^{*}\right)=\inf _{u \in P_{m}} \varphi(u) \leq \sup _{u \in \partial B_{a_{n}}} \varphi(u)
$$

From the inequality above and the result (d) of Step 2, one gets

$$
\varphi\left(u_{m}^{*}\right) \rightarrow-\infty \quad \text { as } m \rightarrow \infty
$$


By the conclusion of Step 3, one has $\bar{u}_{m}^{*} \neq b_{m}$ for large $m$. From this one deduces that $u_{m}^{*}$ is an interior point of $P_{m}$ and $u_{m}^{*}$ is a critical point of $\varphi$. Then, the proof of Theorem 1.1 is completed.

\section{Competing interests}

The authors declare that they have no competing interests.

\section{Authors' contributions}

Both authors contributed equally to the writing of this paper. Both authors read and approved the final manuscript.

\section{Acknowledgements}

The authors sincerely thanks the editors and the referee for their many valuable comments, which helped improving the article. This research was partly supported by the Science Foundation of Huanggang Normal University, China (No. 201617503)

Received: 10 July 2016 Accepted: 10 November 2016 Published online: 22 November 2016

\section{References}

1. Guo, ZM, Yu, JS: The existence of periodic and subharmonic solutions of subquadratic second order difference equations. J. London Math. Soc. (2) 68(2), 419-430 (2003)

2. Guo, ZM, Yu, JS: Periodic and subharmonic solutions for superquadratic discrete Hamiltonian systems. Nonlinear Anal. 55(7-8), 969-983 (2003)

3. Guo, ZM, Yu, JS: Existence of periodic and subharmonic solutions for second-order superlinear difference equations Sci. China Ser. A 46(4), 506-515 (2003)

4. Bin, HH, Yu, JS, Guo, ZM: Nontrivial periodic solutions for asymptotically linear resonant difference problem. J. Math. Anal. Appl. 322(1), 477-488 (2006)

5. Guo, ZM, Yu, JS: Applications of critical theory to difference equations. In: Differences and Differential Equations. Fields Inst. Commun., vol. 42, pp. 187-200. Amer. Math. Soc., Providence, RI (2004)

6. Ma, MJ, Yu, JS: Existence of multiple positive periodic solutions for nonlinear functional difference equations. J. Math. Anal. Appl. 305(2), 483-490 (2005)

7. Xue, YF, Tang, CL: Existence of a periodic solution for subquadratic second-order discrete Hamiltonian systems. Nonlinear Anal. 67(7), 2072-2080 (2007)

8. Xue, YF, Tang, CL: Multiple periodic solutions for superquadratic second-order discrete Hamiltonian systems. Appl. Math. Comput. 196(2), 494-500 (2008)

9. Yan, SH, Wu, XP, Tang, CL: Multiple periodic solutions of second order discrete Hamiltonian systems. Appl. Math. Comput. 234, 142-149 (2014)

10. Yu, JS, Guo, ZM, Zou, XF: Periodic solutions of second order self-adjoint difference equations. J. London Math. Soc. (2) 71(1), 146-160 (2005)

11. Zhou, Z, Yu, JS, Guo, ZM: Periodic solutions of higher-dimensional discrete systems. Proc. R. Soc. Edinb. A 134(5), 1013-1022 (2004)

12. Che, CF, Xue, XP: Infinitely many periodic solutions for discrete second order Hamiltonian systems with oscillating potential. Adv. Differ. Equ. 2012, 50 (2012)

13. Tang, XH, Meng, Q: Solutions of a second-order Hamiltonian system with periodic boundary conditions. Nonlinear Anal. 11, 3722-3733 (2010)

14. Mawhin, J, Willem, M: Critical Point Theory and Hamiltonian Systems. Applied Mathematical Sciences, vol. 74. Springer, New York (1989) 